\title{
Synchrotron Micro Tomography Reveals 3D Shape of Precipitates in Cast Magnesium Alloy
}

\author{
Klaus-Dieter Liss ${ }^{1}$, Xavier Thibault ${ }^{2}$, Huijun $\mathrm{Li}^{3}$ and Phil Bendeich ${ }^{1}$ \\ 1. Australian Nuclear Science and Technology Organisation, New Illawarra Road, Lucas Heights, NSW-2234, Australia \\ 2. European Synchrotron Radiation Facility, B P 220, F-38043 Grenoble Cedex, France \\ 3. Faculty of Engineering, University of Wollongong, NSW-2522, Australia
}

Received: August 08, 2010 / Accepted: August 27, 2010 / Published: February 10, 2011.

\begin{abstract}
Aim of the study is to compare the morphology of inclusions in magnesium alloy, investigated by both computed micro-tomography and light microscopy. Precipitates of denser material are found. The information of the light microscopic studies alone is by far insufficient to conclude on their three-dimensional shape. In contrast, the tomography reveals the full topology in all dimensions. The described inclusions are flake-like and can extend with high aspect ration into one, two or three dimensions.
\end{abstract}

Key words: Synchrotron radiation, computed tomography, light microscopy, metallurgy, magnesium.

\section{Introduction}

Magnesium alloy is the lightest of the readily available low density metals with suitable mechanical properties for modern devices where weight plays a role and much research is undertaken to engineer those properties for different applications. This can be done through structural refinement by the addition of additives creating nucleation sites during solidification of cast material [1]. On the other hand, segregation takes place leading eventually to inclusions or precipitates with weak mechanical properties [2-4]. A further problem is the formation of pores when gas cannot escape from the system before solidification [5].

Microscopic imaging using visual light is one of the most widely used techniques in metallography for the investigation of microstructures down to a micrometer scale. The main drawback, however, evolves from accessing only a two-dimensional, well

Corresponding author: Klaus-Dieter Liss (1962- ), male, senior research fellow, research field: neutron and synchrotron X-ray diffraction and materials physics. E-mail: kdl@ansto.gov.au, liss@kdliss.de. prepared surface which shows not more than a cross section of three-dimensional structures. Contingently, the surface can be removed layer by layer in order to obtain a depth information. A similar range of size and resolution can be accessed by 3D computed tomography as presented here.

\section{Experiment}

\subsection{Sample Preparation}

For the present investigation, we used cast magnesium alloy of composition 96Mg-3Al-1Zn (at.\%). The material was produced by direct chill (DC)-casting in an industrial process (Magnesium-Elektron Limited, MEL) and received a solution heat treatment for $12 \mathrm{~h}$ at $350^{\circ} \mathrm{C}$. This is a typical procedure to prepare billets for subsequent deformation processes such as extrusion or rolling. A master block of $28 \times 26 \times 80 \mathrm{~mm}^{3}$ was cut out of the bulk from which all other samples were machined subsequently. A polished surface for optical microscopy of $20 \times 16 \mathrm{~mm}^{2}$ was prepared from a dedicated cut and a lengthy cylinder with $1.4 \mathrm{~mm}$ diameter spark eroded from the vicinity. 


\subsection{Light Microscopy}

The optical micrograph Fig. 1 of $500 \mu \mathrm{m}$ bottom edge length shows in the top part the gray level image as acquired from the microscopy sample. The bottom Fig. 1 shows the same image after treatment with the public domain NIH Image program ImageJ $[6,7]$ where background has been removed by the rolling-ball method with radius 200 pixels and subsequent adoption of gray levels for higher contrast. Fluctuations in the light gray background indicate the network of grain boundaries. Attention is drawn to the dark appearing structures, such as hollow pores, which appear as round blobs and elongated structures of up to $80 \mu \mathrm{m}$ lengths as in the center and the bottom of the image. These features will be identified as hollow pores and elongated inclusions. The images look


Fig. 1 Visual light micrographs of the polished magnesium alloy, as recorded (top) and after image treatment (bottom). qualitatively similar to earlier findings [8]. On finer length scales strings of sub-micrometer dots arranged in straight lines exist, which are not further discussed here.

\subsection{Synchrotron Micro-Tomography}

Three dimensional computed micro-tomographies on the cylinder sample have been undertaken at the beamline ID19 at the ESRF. The micro-tomography apparatus installed at this beamline takes the advantages of the homogeneous, parallel, monochromatic, and highly coherent, very high photon flux produced by the synchrotron. The mode of operation was absorption-contrast. The detection device gathered a shutter controlling the exposure time, a fluorescent screen converting the X-rays to visible light, an optical system and an adapted detector. The sample was centered accurately with the cylinder axis parallel to the instrument axis of the high precision rotation stage. The transmitted synchrotron X-ray beam of $13 \mathrm{keV}$ was recorded by a $2048 \times 2048$ pixel FReLoN camera [9] with a field of view of $1433 \mu \mathrm{m}$ as a function of sample rotation. A total of 1200 radiographies, obtained from 0 to 180 degrees with 0.15 angular degree increment, were required to obtain a usable reconstructed volume. A filtered back projection algorithm was used to retrieve the 3D map of absorption value from the set of the 2D radiographies. For this purpose, the HST package for high speed tomography based on Fourier inversion was used [10]. The resulting volume data is about 8.5 GB. Rendering of the 3D map of absorption and segmentation were done through the free software packages Image [6, 11] and the plugin Volume [7]. Using a linear relationship the absorption values are translated into gray values.

\section{Results and Discussion}

A section of a reconstructed volume slice perpendicular to the cylinder axis is given in Fig. 2, revealing dark, elongated inclusions, proving 


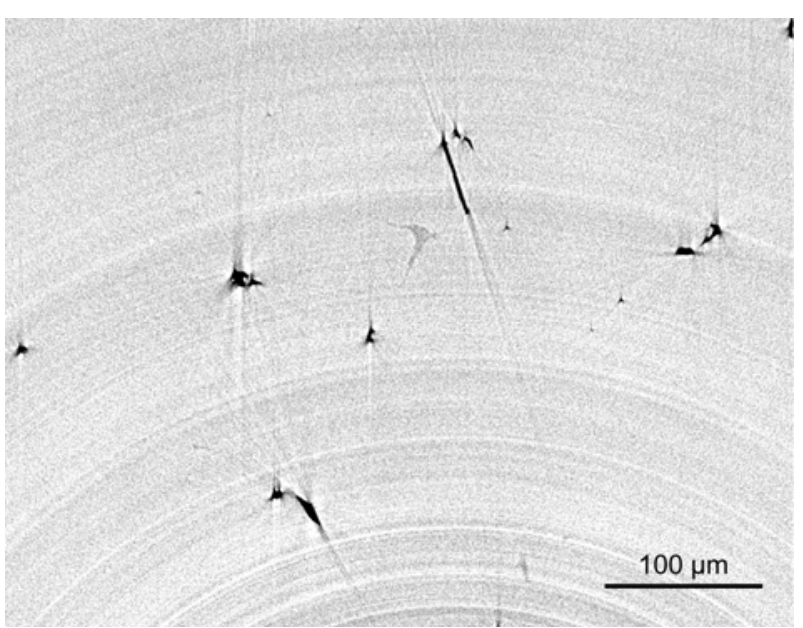

Fig. 2 Slice through the reconstructed volume stack from the 3D computed tomography. The features of elongated precipitates are qualitatively similar to those in Fig. 1.

consistency to Fig. 1. Light gray concentric rings and streaks are artifacts from the process of acquisition and reconstruction. From the radiograms and in contrast to lighter appearing regions which have been identified as pores (Fig. 3), the darker contrast of the inclusions indicates higher X-ray absorption and thus precipitation of material with higher atomic number than the Mg matrix alloy. However, it cannot be told so far, whether these precipitates are needle-like or extend into further dimensions.

Much more information is obtained by projections of the volume data into top, side and front view displayed in Fig. 3, where the average gray levels projected through the whole volume section are shown. The morphology of the inclusions looks significantly different than in Figs. 1 and 2 and reveal that the elongated structures are extended and sometimes linked in knots. Further, light gray blobs appear in the upper right hand corner which are pores, i.e., hollow voids in the metal with less $\mathrm{X}$-ray absorption along the lines of view. The projection is a good representation to obtain an overview into the whole volume, however, according to the total thickness of the projection, more or less background fluctuations accumulate.

Three dimensional rendering of iso-graylevel surfaces are presented in Fig. 4 for different views after successive rotation of the top view in $30^{\circ}$ steps around
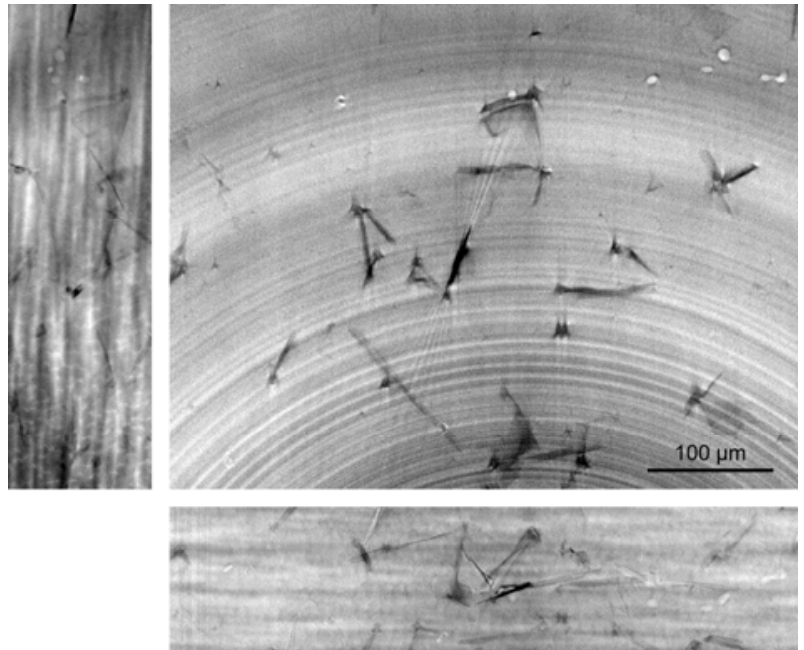

Fig. 3 Projections trough the cropped volume stack along all three Cartesian dimensions, i.e. side view (left), top view (center) and front view (bottom). Eutectic inclusions appear dark and pores light.
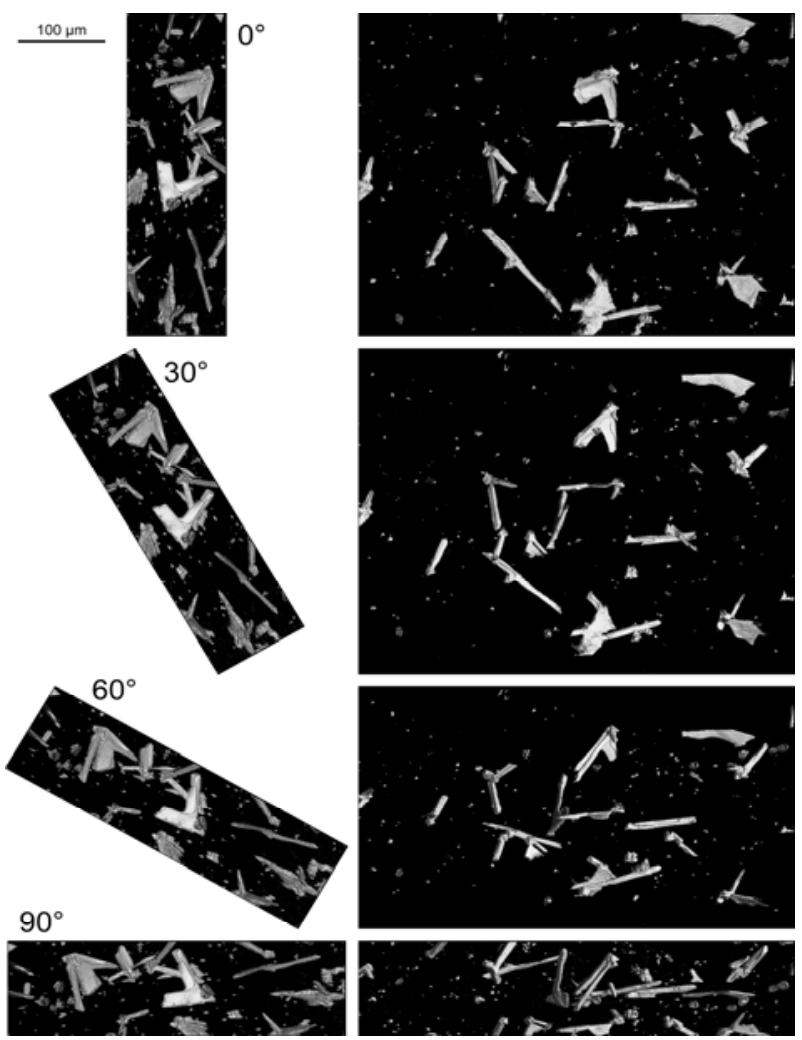

Fig. 4 Rendered volume showing the eutectic inclusions only-not the matrix nor the pores. The rows show different views rotated stepwise by $30^{\circ}$ from side and top view (first row) to side and front view (last row).

a horizontal axis. The $0^{\circ}$ and $90^{\circ}$ views correspond to the projections in Fig. 3. The inclusions are rendered lighter gray and can be distinguished from the fully 
transparent matrix, appearing black in the figure. Individual objects move differently through the individual figures of view due to the full three-dimensional character of the data.

The denser precipitates appear either as 2D thin sheets of micrometer thickness or 1D rods slightly thicker. Each object can now be cropped from the large volume and rendered independently in order to find out its full extensions under individual angles of view, some of them even extending the $100 \mu \mathrm{m}$ mark. The objects are quite flat and straight in their sub-dimension and often linked together at knot points where they change orientation abruptly by a large angle. They combine to flake-like structures remembering some crystallographic orientations and regularity behind and it is likely that they are oriented between the grains of the Mg matrix. It may happen that the precipitates are not just constrained by a 2D interface between two neighboring grains, but by a triple junction which results then in the precipitates extended in 1D. Assuming a typical given volume of a precipitate, the observation is consistent with the larger thickness of the $1 \mathrm{D}$ as compared to the $2 \mathrm{D}$ form. It appears as well that some 2D sheets have one thicker edge, that is, where the grain boundary face meets a corner. The observed knots are just corners of multiple grains joining together in a triple junction.

Nucleation and growth of $\mathrm{Mg}$ alloy grains takes place during the solidification of the cast process. As the crystallites grow, chemical segregation occurs raising the concentration of heavier elements in the remaining liquid and forming eventually an eutectic, such as $\mathrm{Mg}-\mathrm{Mg}_{17} \mathrm{Al}_{12}$ [12], which is then squeezed between the regular shaped $\mathrm{Mg}$ grains, solidifying at much lower temperatures. The size and distribution of those precipitates is most important for the resulting mechanical properties of the material [2-4].

\section{Conclusions}

Precipitates of denser material have been characterized in 3D, which probably form between the grains of magnesium material. They show rod, plate and flake like morphology, depending whether they occur between corners, faces and triple junction grain boundaries. Aspect ratios can be high with thicknesses in the $1 \mu \mathrm{m}$ range and lateral extension $10 \mu \mathrm{m}$ to $100 \mu \mathrm{m}$.

As an outlook, future investigations could reveal the evolution of such inclusions during thermo-mechanical processing, in particular under in-situ conditions [13]. Further, synchrotron sources and techniques evolve rapidly with resolution down to the nanometer scale or high-energy X-rays with better penetration into heavy or thick material [14].

\section{Acknowledgments}

The authors wish to express many thanks to Dr. Dietmar Letzig and Dr. Jan Bohlen from the GKSS research center, Germany for providing the samples and discussion and to Dr. José Baruchel, ESRF, Grenoble for granting test time on his beamline.

\section{References}

[1] M. Bamberger, Structural refinement of cast magnesium alloys, Materials Science and Technology 17 (2001) 15-24.

[2] Q.L. Jin, J.P. Eom, S.G. Lim, W.W. Park, B.S. You, Grain refining mechanism of a carbon addition method in a Mg-Al magnesium alloy, Scripta Materialia 49 (2003) 1129-1132.

[3] T. Laser, M.R. Nürnberg, A. Janz, C. Hartig, D. Letzig, R. Schmid-Fetzer, R. Bormann, The influence of manganese on the microstructure and mechanical properties of AZ31 gravity die cast alloys, Acta. Materialia 54 (2006) 3033-3041.

[4] M. Marya, L.G. Hector, R. Verma, W. Tong, Microstructural effects of AZ31 magnesium alloy on its tensile deformation and failure behaviors, Materials Science and Engineering A 418 (2006) 341-356.

[5] S.G. Lee, A.M. Gokhale, Visualization of threedimensional pore morphologies in a high-pressure die-cast Mg-Al-Re alloy, Scripta Materialia 56 (2007) 501-504.

[6] M.D. Abramoff, P.J. Magelhaes, S.J. Ram, Image processing with ImageJ, Biophotonics International 11 (2004) 36-42.

[7] M.D. Abramoff, M.A. Viergever, Computation and visualization of three-dimensional soft tissue motion in the 
orbit, IEEE Transactions on Medical Imaging 21 (2002) 296-304.

[8] M. Bamberger, Phase formation in Mg-Sn-Zn alloys-thermodynamic calculations vs experimental verification, Journal of Materials Science 41 (2006) 2821-2829.

[9] J.C. Labiche, O. Mathon, S. Pascarelli, M.A. Newton, G.G. Ferre, C. Curfs, G. Vaughan, A. Homs, D.F. Carreiras, The fast readout low noise camera as a versatile $\mathrm{X}$-ray detector for time resolved dispersive extended $\mathrm{X}$-ray absorption fine structure and diffraction studies of dynamic problems in materials science, chemistry, and catalysis, Review of Scientific Instruments 78 (2007) 091301.

[10] A.P. Hammersley, HST: high speed tomography, ESRF , Grenoble, France (1999-2001), available online at: http://www.esrf.eu/computing/scientific/HST/HST_REF/ hst.html.
[11] W.S. Rasband, ImageJ, National Institutes of Health, Bethesda, Maryland, USA (1997-2008), available online at: http://rsb.info.nih.gov/ij/.

[12] J.L. Murray, Al-Mg (aluminium-magnesium), Phase Diagrams of Binary Magnesium Alloys, 1988.

[13] K.D. Liss, A. Bartels, H. Clemens, S. Bystrzanowski, A. Stark, T. Buslaps, F.P. Schimansky, R. Gerling, C. Scheu, A. Schreyer, Recrystallization and phase transitions in a gamma-TiAl based alloy as observed by ex- and in-situ high-energy X-ray diffraction, Acta Materialia 54-14 (2006) 3721-3735.

[14] K.D. Liss, A. Bartels, A. Schreyer, H. Clemens, High energy X-rays: A tool for advanced bulk investigations in materials science and physics, Textures and Microstructures 35 (2003) 219-252. 